\title{
ABDULLA QODIRIYNING O`ZBEK ADABIY TILI VA MATBUOTI RIVOJIDAGI XIZMATLARI
}

Murodqosim ABDIYEV*

\begin{abstract}
Annotatsiya
Ushbu maqolada o'tgan asrning boshlarida Turkistonda ro`y bergan siyosiy va ma rifiy o'zgarishlarning qisqacha sharhi berilgan. Shu bilan birga musulmon turkiylar jadidchiligining asoschisi Ismoil G‘aspiralining “ Tarjumon” gazetasining o'lkada ma rifatchilik g'oyalarining yoyilishidagi roli bilan bo'liq masalalari ham ko'rsatib o'tilgan. Shuningdek maqolada o'sha davrda o'zbek adabiyotining rivojlanishiga salmoqli hissa qo'shgan, o'zbek romanchiligining asoschisi Abdulla Qodiriyning yangi o'zbek adabiy tili, xususan o'zbek milliy matbuoti rivojidagi xizmatlari ham yoritib berilishiga harakat qilingan. Xususan, uning yangi o'zbek adabiy tili hamda milliy matbuoti shakllanishidagi xizmatlari, aniqrog $i \mathrm{uni}$ yaratish borasidagi harakatlari va zahmatlari dalillar bilan ifodalangan. Uning hozirgacha bir nom bilan o'zgarishsiz nasr etib kelinayotgan “ Mushtum” jurnalining tashkilotchilaridan biri ekanligi, adib jurnal sahifalarida mahalliy matbuotda yagona o'zbek adabiy tili me`yorlarining shakllanishiga putur etkazayotgan unsurlarni ayovsiz ravishda hajv qilish jarayonlari tahlil etilgan. Shunigdek, maqolada adibning poliglot sifatida arab, fors, turk, rus tillarini mukammal bilganligi bois juda ko'plab adiblarning asarlarini o'zbek tiliga tarjima qilganligi hamda sharqshunos olim K. Yudaxin bilan hamkorlikda “ Ruscha-ozbekcha lug'at” tuzish ishlarida qatnashganligi singari masalalar haqida ham fikr yuritilgan.
\end{abstract}

Kalit So`zlar: Jadidchilik, Adabiy Til, Matbuot, So`z, Gazeta, Lug`at, Tarjimon.

\section{ABDULLA KADİRI'NIIN ÖZBEK EDEBî DİLINII VE BASININI GELISTTIRME YÖNÜNDEKİ HİZMETLERİ \\ Özet}

$\mathrm{Bu}$ makale, geçen yüzyılın başında Türkistan'da meydana gelen siyasî ve eğitimsel değiş̧imlere kısa bir bakış sunmaktadır. Aynı zamanda, Müslüman Türk yoğunluğunun kurucusu İsmail Gaspıralı'nın ülkedeki aydınlanma fikirlerinin yayılmasında "Tercüman" gazetesinin rolünden de bahsedilmiştir. Buna ek olarak, makalede o zamanlar Özbek edebiyatının gelişimine önemli bir katkı sağlayan Özbek romanının kurucusu Abdulla Kadiri'nin yeni Özbek edebî dilinin, özellikle de Özbek ulusal basınının gelişmesi ile ilgili eserlerine de değinilmiştir. Özellikle, yeni bir Özbek edebî dilinin ve ulusal basının oluşumundaki hizmetleri üzerinde de durulmuştur. Şimdiye kadar adı değişmeden neşredilip günümüze kadar gelen "Muştum" dergisinin organizatörlerinden birisi olması dolayısıyla, onun bu derginin sayfalarında ve yerel basında standart Özbek dilinin standartlaşmasına engel olan unsurları eleştirdiği yazılar incelenmiştir. Ayrıca makalede edibin Arapça, Farsça, Türkçe ve Rusçayı bir poliglot olarak mükemmel bir şekilde bilmesi, birçok yazarın eserini Özbekçeye tercüme etmesi ve şarkiyatçı bilgin K.Yudahin ile beraber "Rusça-Özbekçe Lügat" hazırlama gayreti gibi konulara değinilmiştir.

Anahtar Sözcükler: Cedidcilik, Edebî Dil, Basın, Söz, Gazete, Sözlük, Tercüman.

\footnotetext{
*Prof. Dr., Semerkant Devlet Üniversitesi, el-mek: murodkasim63@mail.ru
} 


\title{
ABDULLA KADIRI UZBEK LITERARY LANGUAGE AND PRESS DEVELOPMENT SERVICES
}

\begin{abstract}
Annotation
This article provides a brief overview of the political and educational changes that occurred in Turkistan at the beginning of the last century. It was also mentioned the role of the newspaper "Tarjumun" in the spread of Enlightenment ideas in the country by Ismail Gaspirali, the founder of the Muslim Turkic density. In addition, the article made an important contribution to the development of Uzbek literature at that time, while the works of Abdulla Kadiri, founder of Uzbek novels, were also highlighted in the development of the new Uzbek literary language, especially the Uzbek national press. In particular, his services in the formation of a new Uzbek literary language and the national press, or rather his efforts and efforts to create it, are represented by evidence. Having been one of the organizers of the magazine "Mushtum", which has remained with the same name invariably until now and his bitter criticism towards factors which has damaged the formation of criteria of standard Uzbek language in the pages of magazine were analyzed. In the article, some ideas were put forward about his knowledge of Persian, Arabic, Turkish and Russian as a polyglot, translating a lot of authors works to Uzbek language and collaboration with orientalist scientist K. Yudakhin to prepare Uzbek-Russian Dictionary.
\end{abstract}

Key Words: Jadidism, Literary Language, Mass-Media, Word, Newspaper, Dictionary, Translator.

Turkiy tillar ichida o'ziga xos mavqega ega bo'lgan ona tilimiz taqdiri tarix zarvaraqlarida bitilgan unutilmas olamshumul voqealarning ayanchli asoratlari va sezilarli ta`sirlarinining ma`lum bir holatlarini o'zida mujassam etgan mukammal til hisoblanadi. Eronliklar, yunonlar, arablar, mo'g'ullar va ruslar bosqining oqibatida ham ma`naviy ham iqtisodiy tomondan yo'qotishlar bo'ldi. Turk hoqonligi, somoniylar, qoraxoniylar, temuriylar, shayboniylar, xonliklar, sho'rolar tuzumi boshqaruvlarida esa ma lum bir bosqichlarni bosib o'tdi. Ya`ni ona tilimiz leksikasi, fonetikasi, grammatikasi, uslubshunosligida jiddiy o'zgarishlar yuz berdi.

Mustaqillik esa ona tilimiz rivoji uchun keng imkoniyatlar eshigini ochib berdi. Xususan, O‘zbekiston Respublikasi Prezidentining 2019-yil 19-oktabridagi “O'zbek tilining davlat tili sifatini, nufuzi va mavqeni tubdan oshirish chora-tadbirlari to'g'risida"gi PF -5850sonli Farmoni ijrosini ta`minlash maqsadida 2020-2030 yillarda o'zbek tilini yanada rivojlantirish hamda til siyosatini takomillashtirish kosepsiyasi ishlab chiqilganligi ona tilimizning yanada takomillasuviga hamda rivojlanishiga keng imkoniyatlar eshigini ochib berdi.[1.]

Tarixdan ma limki, temuriylar davridan keyin tanazulga uchragan o'lkaning ijtimoiysiyosiy hayotida hamda iqtisodida jiddiy o'zgarishlar bo'lmadi, hisob. Jamiyatda hukm surgan boshboshdoqlik xonliklar o'rtasidagi o'zaro nizo va to'xtovsiz urushlar xalqning tinkasini quritib, Turkiston o'lkasini qoloqlik va jaholat botqog'iga torta boshladi [ 2. 17].

$\mathrm{XX}$ asr boshlariga kelib esa musulmon turkiylarning jadidchilik harakati asoschisi Ismoil G'aspirali g'oyalari butun turk dunyosi olamiga tarqala boshlagan edi. "Jadidizm harakatining asosiy maqsadi islom shariati asosini saqlagan holda Yevropa madaniyatining ibratli tomonlarini kiritish orqali Turkiston o'lkasi, Buxoro amirligi va Xiva xonligini aholisini qoloqlikdan, razolat botqog'i singari illiatlardan qutqarishga barobarida milliy masalalarni hal qilishga qaratilgan edi. Shu bois ham ular oldida jadid maktablari ochish, xayriya jamg 'armalari tuzish, matbaa ishlarini rivojlantirish, kutubxona, kitobxonlik, matbuot, adabiyot va teatr san`ati singari sohalar ishlarini yo‘lga qo‘yish sigari vazifalar turar edi”[3., 4.]. 
Turkistonda jadidchilik g'oyalarini aholi o'rtasida keng tarqatish va targ'ibot tashviqot qilishda tatar ziyolilarining xizmatlari ham katta bo'ldi. Ana shunday ma rifatparvarlardan biri Ismoil Gaspirinskiy( 1885-1914) edi[ 4.].

$\mathrm{Bu}$ davrga kelib, "musulmon turkiylar jadidchilik harakatining asoschisi Ismoil G'aspirali va uning tashabbusi bilan nashr etilgan "Tarjumon” gazetasi materiallaridagi tashviq va ta sirlar tufayli o'lka jadidlari tomonidan Turkistonni razolat botqog idan chiqarish uchun xalqni ma`rifatli qilish ...” [5.,45] singari g`oyalarni amalga oshirish uchun bir qator islohatlar o‘tkazilishiga harakat qilindi.

Xususan, "Ismoil G‘aspirali 1908-yilda Turkistonda bo‘lib ilg‘or ma`rifarparvarlardan muftiy Mahmudxo‘ja Behbudiy, A. Shakuriy va boshqalar bilan uchrashdi, o'zbek, tojik, tatar bolalari uchun maktablar ochib jadidchilik g'oyalarini keng tarqatdi. Bu g'oyalarni tarqatishda Lsmoil Gaspirinskiyning o'zi tashabbus ko'rsatib chiqargan “Tarjimon”ning o'rni katta bo'ldi. U musulmon milliy ozodlik harakatining yo'lboshisi sifatida tanilgan edi. "Dor ul rohat musulmonlari” ilmiy-fantastik asari, “ Yuz yildan so'ng 2000-sana” badiiy publitsitik roman, "Turkiston ulamosi” kabi kitoblar uning qalamiga mansub edi”[ 6. 285 ]. Uning o'lka ziyolilari bilan uchrashishi musulmonlar hayotida muhim o'zgarishlarni boshlab berdi.

Sir emaski, XX asr boshlarigacha bo'Igan davr adabiy muhiti vakillari ijodida Alisher Navoiy asos solgan eski o'zbek adabiy til(chig‘atoy tili) an`ana va ta`siri kuchli edi. Yaratilayotgan asarlar aksariyati adib asarlariga hamohang tarzda yoki uning asarlariga naziralar shaklida yozilar edi. Lekin jamiyat hayotida bo'layotgan o'zgarishlar vaqtli matbuot va kundalik ehtiyojlarni ifodalash maqsadida ommaning og'zaki so'zlashuv nutqiga rus, turk va boshqa tillardan juda ko'plab yangi so'z va terminlar kirib kela boshlayotgan va o'rnasha boshlayotgan edi. Ushbu omillar natijasida xalq ichida adabiy til bilan so'zlashuv tilini yaqinlashtirishga bo'lgan ehtiyoj sezila boshlangan edi.

Bu davrda ma`rifatparvarlik oqimida qalam tebratgan Muqimiy, Zavqiy, Furqat, Avaz O‘tar, Sattorxon, Turdi singari adiblar asar mazmun va shaklining o'ziga xos xususiyatlarini ochib berishda rus, turk va boshqa tillarga xos lug'aviy qatlamlardan mohirlik bilan foydalanishga harakat qilsh jarayonlari kuzatila boshlandi. Asta-sekinlik bilan ma`rifatparvar ziyolilar "Turkiston viloyatining gazeti” sahifalarida jamiyatda ro'y berayotgan o'zgarishlarga munosobat bildirilgan maqolalar paydo bo'la boshladi. Asta-sekinlik bilan o'zbek matbuotchiligi va uning uslubi shakllana boshladi. Truppa shaklidagi xalq teatrlari tashkli etila boshlandi. Samarqanda birinchi marotaba o'zbek tilida Mahmudxoja Behbudiyning” Padarkush” drammasi sahnaga qo“yildi. Ma`rifatparvarlik yo'nalshlari bilan bir qatorda umumturkiy(turk tili) tilni targ'ib qilsh nazariyalari ham paydo bo'la boshladi. Ikkinchi tomonidan esa sho'rolar tomonidan turkiylarni bir-biriga yaqinlashtirmaslikka pinhona harakatlar bo'la boshladi. Jumladan, o'zbek millatini bo'laklarga bo'lish maqsadida har bir hududning o'z hududiy tilini yaratish va unda badiiy asarlar hamda darsliklar yozishga da vat etish g'oyalari goh yashirincha, goh oshkora targ'ib qilina boshlangan edi. Bu esa ziyolilar oldiga ikki yo'Idan birini tanlash masalasini ko'ndalang qo'yilayotgan edi.

Umumturkiy til yaratish nazriyasi ilk marotaba qrim tatarlari donishmandi Ismoil G'aspirali o'zi tashkil etgan “Tarjumon” gazetasi orqali targ ‘ib qildi. Shundan so'ng 1905- yilda Butunrossiya musulmonlarining qurultoyida uning tashabbusi bilan turkiy tilli millatlar maktablarining yuqori sinflarida ygona turkiy til, ya`ni turk tilining o'qitilishi masalasi o'rtaga qo'yildi.

Turkiston jadidlari uchun "Tarjumon” gazeta bilim olish manbai bo'lib xizmat qila boshladi.”... Ular Ismoil Gaspirinskiy faoliyatini belgilab berilgan mazkur gazetani o'lkada yangi usuldagi ta limning yoyilishida hamda jamiyatga xos kasalliklarni yo 'qotishda ommaga em bo "lishini tushunib yetdilar"[ 7., 309]. Bevosita "Tarjumon" gazetasida targ'ib qilinayotgan g'oyalar ta sirida "Taraqqiyot”, "Xurshid”, "Shuhrat”, “Tujjor”, "Sadoi Farg'ona”, "Al isloh” singari gazetalar hamda "Oina” jurnalini tashkil etildi. Lekin ular turli hududlarda joylashgani 
bois ularda endi shakllanayotgan hozirgi o'zbek adabiy tili me`yorlariga rioya qilish, xususan, matbuot tili va uslubi masalalarida har xilliklar mavjudligi oqibatida tushunmovchilk va anglashilmovchiliklarning yuzaga kelishiga olib kelayotgan edi.

Bu davrga kelib, aniqrog'i o'tgan asrning 20- yillarida muomalada qo'llangan arabchaforscha so'zlarning muqobili sifatida matbuot va og'zaki so'zlashuv nutqi orqali ruschabaynalmilal so'zlar kirib kela boshladi. O‘zbek ziyolilari, xususan, jadidlar ularga puristik yo‘l tutishni nazarda tutgan holda vaqtli matbuot, badiiy asarlar hamda ayrim risola va lug'atlarda ularning o'rnida o'zbekcha(turkiycha) variantlarini qo'llash tavsiya etishdi: proletar-yo'sil, poezd- otasharava, samovar- o'zi qaynar, xlopkom-samovar, fontan- qaynar quduq, traktor- o't omoch va hakoza.

Ana shunday bir davrda o'zbek jadid adabiyotining bir qator ziyolilari qatori atoqli adib Abdulla Qodiriy ham bu jarayondan chetda turmadi. U “Mushtum” jurnali tashkilotchilaridan biri sifatida uning sahifalarida matbuot tilini so'zlashuv tiliga yaqinlashtirish orqali shakllanayotgan o'zbek adabiy tilini takomillashtish tashabbusi bilan chiqdi va o'z ijodiy faoliyatida bunga amal qila boshladi. Adibning bundan maqsadi yangi tashkil etilayotgan maktablarda ona tilini o'qitilishi istagida bo'lgan ziyolilarni qo'llab- quvvatlash maqsadida endigina shakllanayotgan hozirgi o'zbek adabiy tilining me`yorlashuvini ta`minlash hamda uning buzilishiga qaratilgan turli xil unsurlardan tozalash ishlariga ma`lum darajada hissa qo'shish niyatida edi.

"Mushtum” jurnali sahifalarida adibning bu masalaga bag‘ishlab, jonkuyarlik bilan bayon etgan qator hajviy maqolalari bosilib chiqa boshladi. Ushbu o'rinda adibning o'zi aytgan quyidagi fikrlarni keltirishni o'rinli deb hisoblaymiz: "Yozganing agar o'zingni yig'latmasa kuldirmasa, o'zgani ham hech yig'latmas-kuldirmas...”[8., 24].

Biz quyida adibning matbuot tili va uslubi haqidagi ayrim fikrlariga munosobat bildirishga harakat qilamiz:

Jumladan, adib “Qizil O‘zbekiston” gazetasining 182- sonida bosilgan "Sholi ekishni kuchaytirish yo 'lida” nomli maqola "Hozirgi vaqtda O‘zbekistonda erga sholi ekiladi....” deb boshlanadi. Biz "yerga sholi ekiladi..." degan jumlani o“qib judayam hayron qoldik. . O‘yladikim, ilgari "osmonga sholi ekilar ekanmi!? Har holda bu ham "yangi kashfiyotlardan” bo'lsa kerak, deb bilganmiz[9.].

"Bo bunisi kim?- Pirkurlor... - bu nima nomoqulchilik!.. kulolarning piri-ya, hech balo ursin sani, sango aslo vahim degan narsa bormi ahi!... Kulollarning piri hazrati Shis alayhissalom bo'ladi-yu, sani buni pirkurlor deysan[9.].

So'roqlar: "Olimpiyoda” degan so'zdan nimani tushunasiz?

Javoblar: "Olimpiyoda” -Olim akam piyoda yuradi, Olim akam piyoda boradi, Olim akam piyoda turadi[9.] va hakoza.

Shunigdek jurnal sahifalarida adib satirik maqsadlarda ba`zi terminlarning tuzilishini parodiya qilish asosida hajviy so`z va so`z birikmalari hosil qilingan. Quyidagilarga e`tibor bering:

Xulinologiya. $\mathrm{Bu}$ so‘z tuzilish jihatidan biologiya, geologiya, filologiya singari terminlarga oxshatma tarzda hosil qilingan. Shahrimizda bo 'Igan "Xuligonologiya” institutining birinchisi deb O'rdani tanidim[9.]. Bu yerda Toshkentning O'rda dahasi xuligan - bezorilar makoniga , ular ta`lim olib chiqadigan joyga aylantirganligini obrazli qilib tasvirlashda foydalanilgan.

O‘jarizm. O‘jar asosiga -izm affiksini qo‘shish bilan hosil qilingan bu so‘zni “Ozod Buxoro” gazetasi ba`zi islohotlarni yozishda umumiy orfografik qoidalarga rioya 
qilinmayotganligini tanqid qilishda foydalanib shunday yozgan: Hozir “Ozod Buxoro” ba`zi bir islohotlarni "o`jarizm” qonuniga bo ‘ysundirib yoza boshladilar[9.].

Yozuvchining Julqunboy taxallusi bilan "Kalvak mahzunning xotira daftarlaridan" nomli turkum hajviy hikoyalarida, xususan, Abu Chumboq imzosi bilan berilgan "Bir suhbatda..." nomli imzosiz bosilgan "Mavlaviy po‘stunning sarguzashtlari” va "Ayb har du jiyanda erkan" kabi hajviy asarlarida eski ta lim usulida ta lim beruvchi domlo imomlarning kulgili obrazlarini tasvirlagan. Ular nutqida o'quvchilarga tushunarsiz bo'lgan jargon hamda ko'plab fors-tojikcha va arabcha diniy eskirgan so'z va iboralarni qo'llanish orqali satirik obrazlar yaratishning o'ziga xosligi yozuvchi tomonidan mohirona tasvirlab berilgan. Asosan ruhoniylar nutqiga xos bo`lgan asnoyi xurak, ba`daz, alg‘araz, allomai zamon, aknun, tag'yir, tamzir, zabh, az qiyo, botil, bakurra, bachiza, qazora singari eskirgan arab, fors tillariga xos so zlar hamda o'sha tillar va o‘zbek tili elementlari asosida vujudga kelgan kalkalashtirilgan jargonlar: po'shida qimoq, fahmida qilmmoq, furuxt aylamoq, tarsida bo'lmoq, aftida bo'lmoq, nash `u-namo qilmoq, girexta qilmoq singarilarning ma lum bir hududga xosligi tufayli oddiy xalq uchun tushunarsiz bo 'lishi barobarida yangidan shakllanayotgan adabiy til me`yorlarining buzilishiga olib kelishi yozuvchi tomonidan o‘z vaqtida zukkolik bilan payqab olingan. Shu bois adib ulardan kulguli obrazlarni yaratishda mohirlik bilan foydalangan.

Mavlon po‘stun nutqidan: “ Asnoyi zabh, ya`ni xatna zamonida atrofga yig‘ilgan go'daklarning basharti tig'ning zahriga chidaolmay girya og'oz qilsam qahqaha qilishlaridan behad xavotirda erdim. Bag‘dazun osh xitomiga yetub, faqirni chin o'rniga olib bordilar[9.].

Kalvak Maxzum tilidan: "O`zbekiston jumhuriyatida yashaydurg'on butun ag'niyo, anqiyo, azkiyolarga va menga yaqin bo'lgan ulamo. Fuzalo ham dorilfunoda islohohati, zamin, amlak va doxod nalogidan bezori jon bo'lg'an ahli tijoratlarga yetib andog' ma'lum ravshan bo 'lg‘aykim,... ul dunyosi g'affordan ko'ch aylab, xonai oxiratka, ya`ni qabrga keldik[9.].

“ Toshpo'lat tajang nima deydur?” da esa yozuvchi asar qahramonlarining nutqini individuallashtirish maqsadida jamiyat uchun foydali mehnat qilmaydigan qimorboz, maishatparastlar leksikoniga xos jargon va argolardan unumli foydalanadi. Choychaqasini undan undan-bundan qilib tarallasini tortib yurgan Toshpo'latning yetti yarim so'lkavoyini tag'in qaysigo'rso'xdan olsin-a, bobog'imni sotib betayinmi? Yoki sayratmamnimi?... Xudo haqi galavam aynadi. Nima dermasam, mani osdirarmidi, shu xezlaring? Sattor! Bir tiyin bersam... [9.].

Qimorboz: - "lato” bilan "shar” degan qimorlar bir ham yeb, "to‘rtoshiq” yana tarqab kesa, Salim polvonning to'rt aravasi va baloxonaliq imoratini yutib olsam, hamma meliskalar cho'tol oladurg'on bo'lsa...[9.]. Ushbu satrlarda muallif reportyor nutqidagi ruscha hamda eskirgan arabcha va forscha so'zlarning qo 'llanishini yengil kulgi yo'li bilan hajv qiladi.

Shuningdek Abdulla Qodiriy e`lon qilgan asarlarida personaj nutqining qiziqarli bo'lishini ta `minlash maqsadida garmdori(qalampir), bo(yana), qutilish karda(qutilmoq), bacha(bola), go'sht kuydi(somsa), kallapo'sh(do'ppi), jilla(ozgina, picha), ada(ota), chashm(ko'z) singari qo 'llanish doirasi cheklangan so'zlardan unumli foydalangan.

-Hoy to'xta qiling jo'ra, -dedi samarqandlik muxlislardan biri- axiy boshingiz-ku, maynangiz bor deb bilduk. Bo nimaga akun tanangizga o'yla qilasiz?...Yo'q, joningizdan qoqay, jo'ra, gap bundaychangi bo'lmaptur. Buyonda birisi jon kuydirib "tanqid” desun deyam siz istachinalar yel chiqorsa "labbay" deng...

- Hoy, chi bo'lli?

- Ebi(ibi), san og‘zinga qarab gapur! Bo bizni jin urgani yo‘q...[9.].

- Aki, bo tarbuzni yoshuring...

-Bo, qayonga yushurasiz, akun bu sabilni?!...[9.]. 
Birinchi suhbatda shaxsning samarqandlik ekanlgini, buxoroliklarga xos nutqning ikkinchi suhbatda berilganligini bilib olish qiyin emas. Muallif asar personajlarini og zaki nutqqa xos so'zlashuv uslubida gapirtish orqali ularning nutqini individuallashtiribm mahalliy shevalarga xos milliy kaloritni vujudga keltirgan. Shu bilan birga, o'zbek umumadabiy tilida emas, o'gzaki so'zlashuv uslubiga xos bo'Igan shevada so'zlashish nutqini aynan adabiy tilning yozma shaklida qo'llash endigina shaklllanayotgan yangi o'zbek adabiy til me yorlarining buzilishiga olib kelishi mumkinligini kulgi yo'li bilan ko'rsatib beradi va ularni adabiy tilda so'zlashish va yozishga chorlaydi.

Shunigdek yozuvchi asar syujetining ta`sirchan bo 'lishini ta`minlash maqsadida juda ko'plab xalq maqollari va iboralardan unumli foydalanadi. Xususan, u Kalvak Maxzumning jiyani imzosi bilan yozilgan "Toshkent boylari" nomli satirik maqolasida xasis boylardan biri - Hojiga nisbatan xalqning nafrat va g'azabini ifoda etib, shunday murojaat qiladi: " Hoy Hoji! Yoshing anchaga bordi: bir oyog 'ing yerda bo'lsa, bir oyog'ing go'rda: esing borida etangingni yop! Illo, yig'ub terganlaring faqat uch-to'rtta quzg'unlarga qolishini unutma! Oting, doning o'zing bilan birga ko'mulmasin(9.).

Ushbu parchada o'liming yaqin deyish o'rniga “ bir oyog'ing yerda bo'lsa, bir oyog'ing go 'rda” iborasini qo'llash orqali boyning ochko'zligini mohirlik bilan ko'rsatib berilganligini ko'rish mumkin.

Shuningdek, yozuvchining asarlarida " bir cho'qishda qochiradurg'on”, “ qiz ovlamoq”, "boshlarini ikkitadan, oyoqlarini to“rttadan qilib qo“ymoq”, "it eganini qusar”, " ko'rpangga qarab oyoq uzat”, "qoli sust kosibdan, oyog i chaqqon kosib yaxshi”, "Iligi chiroq ko'rmagan”, “ oltin olma, duo ol”, "xotin kishining charxi bilan o'chog'iga tegma”,"yel elkasiga tegdi”, "qanot quyruq berganda bo'ldi”, "tegimizga suv qo ‘yadigan bo'Idi”, “ haddidan oshdi”,”tusini buzmadi”," dunyo mojarosidan qo'l yuvmoqchi", " yuragiga sovuq suv sepmoqchi bo'ldi”, "tutuni ko'kka chiqmoqchi”, "yarasini yordi”, "qo“lini sovuq suvga urmay qo‘ydi”, "xotin kishini ushlab turish”, soyalarida o'ynab-o“ynab yurish” singari ibora va hikmatli so 'zlar o'zbekona hayot va mentalitetni ochib berishda muhim vosita sifatida foydalangan. Birgina "O`tgan kunlar” romani matnida qo'llangan frazeologizmlarning soni 600 dan ortiqligining[10., 9] adibning xalqning ma 'naviy boyligi bo 'Imish iboralardan yaxshi xabardorligidan va ulardan asar mazmunini ochib berishda unumli foydalanganligidan dalolat berib turibdi.

Adib o'zi o'rinli ta kidlaganidek, "orttirmay va kamittirmay, o‘z holicha ko'rsatish”, “yo“qni yo'ndirtirmay, bor gaplarni yozish” kabi ibrotomuz naqllarga amal qildi va bu niyatni amalga oshirish maqsadida asar qahramonlarining nomlarini ham ismini jismiga monand qilib tanlaydi.

Yusufbek hoji, Akram hoji - hammaning nazariga tushgan haj safari amallarini bajarib , hoji unvonlarini olgan kishilar. Oftob oyim - oftobdek istarasi issiq ayol, O‘zbek oyim - o`zbek ayollarining jami hislatlarini o'zida mujassam etgan, peshqadam ayollar vakili. Mirzakarim qutidor - savdo ilmimni yaxshi egallagan ziyoli kishi, Solih mahdum- tajribali va ilmli maktabdor muallim. Obid ketmon- jamoa xo`jaligini tan olmaydigan, mirishkor dehqon. Toshpo'lat tajang - ishi yurishmagan, chani yigit, Kalvak mahzum - kundalik yangiliklardan bexabar, xurofot bilan miyasi g'ovlagan imom. Berdi tatar - adibning Tataristonga qilgan safari taassurotlari natijasida tanlangan jadidnamo, ziyoli kishi. Xatib domla - jomelarda xutba o'quvchi duoxon domla. Anvar - tolei porloq yigit, Ra`no - go'zal va latofatli qiz, Otabek beklar sardori, bekzoda. Kumushbibi - kumushdek aziz va martabali qiz va hakoza. Bu o'rinda yozuvchining qahramonlarga ism tanlashdan maqsadi "ismi-jismiga monand" bo 'lishini ta`minlash niyatida asarning uslubiy xususiyatlariga xos bo'lgan hissiy ta`sirchanlikni oshirishga qaratishdan iborat.

Adib rus, arab, fors, turk, tatar va boshqa tillarni mukammal bilgan. Shu bois o'sha tillarda yozilgan asarlarni originalida o'qigan. U L.Tolstostoy, A. Chexov, M. Gorkiy, A.Tolstoy, U. Xayyom, H. Sheroziy, V. Bedil, J. Rumiy singari adiblarning ijodidan yaxshi 
xabardor bo'lgan. Yatto Misr adibi Jo‘rji Zaydoniyni “ meni roman yozishga havaslantirgan ustoz” deb ulug 'lagan edi.

Jumladan, adib tillar o'rtasidagi aloqa hamda til bilishning ahamiyati va foydali ekanligini ta kidlab shunday degan edi: " Hamma tillar ham qo'shni tillardan "qarz" oladi. Busiz iloji yo'q. Asar faqat $\mathrm{o}^{\prime} \mathrm{z}$ tilidagina yozilsa shirasiz, quruq chiqadi. Yozuvchi o' $z$ xalqining tilini, folklorini kamol o'rganishi shart va bir nechta tillarni bilishi fazilat. Shundagina til boyiydi, asari jonlanadi”[8., 72].“ Bu ayni haqiqat edi. Shu bois ham adib yoshlarga , farzandlariga chet tillarni xususan, rus tilini o'rganishga da`vat etadi. U o'zbek tili ixlosmandlari bo'lgan rus sharqshunoslari E.D. Polivanov, A.I. Andreyev, K.K. Yudaxin va boshqalar bilan til, madaniyat, imlo masalalarida doimiy ravishda o'zaro fikrlashib, hamsuhbat bo'lib turgan. Sharqshunos olim K.K. Yudaxin "O‘zbekcha-ruscha lug'at” tuzish jarayonida Abdulla Qodiriydan doimiy ravishda maslahat olib turgan.

Adib o'zi ham mazkur lug'at tuzish jarayonida qatnashganligi haqida uning o'g'li Habibullo Qodiriy shunday deb yozadi: “ 1933- yilning kech kuzi edi, shekilli, dadam bir-ikki oy bosim o'tirib, “ Ruscha- o'zbekcha to'la so'zli lug'at: ning "P” harfini tuzdilar. Yodimda u kishi sandalda o'tirib, atroflariga o'nlab rus, o'zbek, arab, turk lug'atlarini yoyib qo'yib, alfavit bo'yicha kitoblardan yangi so'zlar izlar va topib yozar, lug'atlardan yangi ma`no qidirar edilar. Nihoyat jadal ishlab, u kishi o'n bir ming so'zli "P” harfli lug'atni shu yilning yoki 1934yilning boshlarida tugatib nashriyotga topshirdilar. Lug'at 1934- yilda lotin alifbosida bosilib chiqdi. Biroq lug‘at haqini olishga kelganda anglashilmovchilik ro ‘y berdi...”[11., 159].

Keyinchalik tuzilgan va nashr etilgan lug‘atlardagi so'zlarning qanday ma`nolarda ishlatilish holatlariga oid faktik materiallar ham asosan Abdulla Qodiriyning "O'tkan kunlar" romani va boshqa asarlaridabn olinganligi keng ilmiy jamoatchilik xabardordir.

Xullas, atoqli adib Abdulla Qodiriy tom ma`nodagi xalqparvar ziyoli sifatida doimiy ravishda o'zbek tilining himoyachisi bo'lgan. U mustabid tuzum amaldorlarining turli vajsabablar bilan turkiy xalqlarning, jumladan, o'zbek millatinig yagona adabiy til ostida birlashishlarini sira ham xohlashmaganliklarini o'z vaqtida anglaganligi bois oqilona ish yuritgan. Adib o'sha davrdagi ijodkorlarni endigina shakllanayotgan yangi o'zbek adabiy tilida, ya`ni hamma o'zbeklar uchun tushunarli bo'lgan shaklda yozishga da`vat etgan va o'zi bunga rioya etgan holda boshqalarga namuna bo'la olgan. Shu ma`noda u o'zbek romanchiligiga asos soldi. Yangi o'zbek adabiy tilida bir qator asarlar yozdi. Nafaqat adabiy til, balki matbuot tili va uslubining shakllanishiga ma 'lum darajada hissa qo'shdi. Hatto lug'atlar tuzish singari ishlarda ham faol qatnashdi. Shu bois uning nafaqat asarlarini, balki hozirgi o'zbek adabiy tili va milliy matbuoti rivojidagi xizmatlarini, xususan, adib asarlari tili va uslubini yanada chuqurroq o'rganish va tadqiq etish filologiya fanining dolzarb masalalaridan biri hisoblanadi. 


\section{Adabiyotlar}

O‘zbekiston Respublikasi Prezidentining 2019-yil 19-oktabridagi “O`zbek tilining davlat tili sifatini, nufuzi va mavqeni tubdan oshirtirish chora-tadbirlari to 'g 'risida”gi PF -5850sonli Farmoni II Xalq so‘zi gazetasi, 2019 -yil, 19- oktabr

ГАФАРОВ, Н. У. (2014). Джадидизм в Средней Азии в конце XIX в. начале XX в. АДД. -Душанбе.

ҚОСИМОВ, Б. (1992) Исмоилбек Гаспиринский. Тошкент.

АБДУАЗИЗОВА, Н. (2000). Туркистон матбуоти тарихи.- Тошкент.

ШАМСУТДИНО, Р.Т., КАРИМОВ, Ш.; УБАЙДУЛЛАЕВ, Ў. (2003). Ватан тарихи. (XVI аср -XX аср бошлари) Иккинчи китоб.- Тошкент :Шарқ.

АБДИРАШИДОВ, 3. (2011). Исмаил Гаспринский и Туркестан в начале ХХ века: связи-отношения-влияние.- Ташкент: Akademnashr.

Қодирийни қўмсаб.- Тошкент: А.Қодирий номидаги халқ мероси нашриёти,1994.

“Муштум” журналининг 1924 -1929 йиллар сонлари.

ЧОРИЕВА, 3.Т. (2000). Абдулла Қодирийнинг “Ўтган кунлари” романидаги мактубларнинг луғавий-маъновий ва услубий хусусиятлари.НДА.-Тошкент.

ҚОДИРИЙ, Х. (1983). Отам хақида.-Тошкент: Ғ.Ғулом номидаги Адабиёт ва санъат нашриёти. 